\title{
Revisiting Goal-Oriented Requirements Engineering with a Regulation View
}

\author{
Gil Regev $^{1,2}$, Alain Wegmann ${ }^{1}$ \\ ${ }^{1}$ Ecole Polytechnique Fédérale de Lausanne (EPFL), School of Computer and \\ Communication Sciences, CH-1015 Lausanne, Switzerland \\ \{Gil.Regev, Alain.Wegmann\}@epfl.ch \\ ${ }^{2}$ Itecor, Av. Paul Cérésole 24, cp 568, CH-1800 Vevey 1, Switzerland \\ g.regev@itecor.com
}

\begin{abstract}
Goal-Oriented Requirements Engineering (GORE) is considered to be one of the main achievements that the Requirements Engineering field has produced since its inception. Several GORE methods were designed in the last twenty years in both research and industry. In analyzing individual and organizational behavior, goals appear as a natural element. There are other organizational models that may better explain human behavior, albeit at the expense of more complex models. We present one such alternative model that explains individual and organizational survival through continuous regulation. We give our point of view of the changes needed in GORE methods in order to support this alternative view through the use of maintenance goals and beliefs. We illustrate our discussion with the real example of a family practitioner association that needed a new information system.
\end{abstract}

Keywords: Goals, Requirements, Regulation, Survival, Appreciative System, Norms, Beliefs.

\section{Introduction}

Since the advent of requirements engineering (RE) research as an academic discipline, its flagship methods have been Goal-Oriented Requirements Engineering (GORE). GORE methods have been lauded as one of the main achievements of the RE community [31, 19]. GORE is still a very active field of research with dedicated workshops and conference tracks (e.g. the $\mathrm{i}^{*}$ International workshop series, International Workshop on Requirements, Intentions and Goals in Conceptual Modeling). Some prominent GORE methods were also developed by RE practitioners, e.g. Goal-Oriented Use Cases [7] and Essential Use Cases [8].

The emergence of GORE methods coincides with a less software centric view of requirements. RE evolved out of software specification methods by capturing more and more of the environment of the envisioned software system [21], i.e. the composite system [31]. GORE methods are based on the understanding that goals justify and explain requirements that are assigned to agents in the composite system (software system and its environment), and that they help detect and resolve conflicts among different stakeholder viewpoints [9]. In GORE methods and subsequently in 
$\mathrm{RE}$, it is assumed that the behavior of the stakeholders in the environment of the software system is predominantly goal-oriented, see for example [16, 21]. Very few $\mathrm{RE}$ researchers have challenged this assumption and there is little debate concerning the epistemological roots of GORE methods

We take Gause and Weinberg's view that RE is about discovering what is desired [12]. In this paper, we show that what people desire has more to do with the way they regulate their affairs than with the goals they pursue. We base our proposal mostly on Vickers' concept of Appreciative System [26, 32, 33]. Vickers believed that goal achievement was not the ultimate explanation for individual and organizational behavior. He argued that [33]:

to explain a doing solely by reference to its intended results would seem to raise insoluble pseudo-conflicts between ends and means, rules and purposes, while it leaves the ongoing activities of normholding with their inherent, ongoing satisfactions hanging in the air as a psychological anomaly called action done for its own sake.

We show that goals are only the visible part of the way individuals and organizations regulate their norms in order to survive.

GORE methods have most of the necessary constructs to model this behavior, e.g. maintenance goals and beliefs. We advocate a more systematic and widespread use of these concepts for better understanding regulation and for improving GORE methods.

For Nuseibeh and Easterbrook there is a type of RE for each end product [21]. They give the following examples: RE for information systems, RE for embedded control systems and RE for generic services e.g. networking and operating systems. In this classification our discussion applies mostly to RE for information systems. In this type of RE, the composite system is the organization that the envisioned computerbased system serves. Nuseibeh and Easterbrook further state that the context of most $\mathrm{RE}$ and software activities is in this field of information systems development. Hence our discussion is applicable to many RE projects.

We explain our proposal with the data we gathered during a recent project we were involved in. The project began with the goal of replacing a spreadsheet (for tracking interns specializing as family doctors) with an on-line application, but it turned out to be about the maintenance of the community of family doctors.

We begin by presenting the family doctor project (Section 2). We proceed by reviewing the relevant GORE research (Section 3). We then introduce the regulation organizational model and show that it can be used as the underlying mechanism, of which goal-oriented behavior is but the visible part (Section 4). We propose improvements to GORE methods based on the regulation-oriented view (Section 5). We review the related work in Section 6.

\section{The Family Practitioner Example}

The project we were involved in was initiated when we were approached by a Family Practitioner (FP) we call Mark. Mark is the president of an association of family 
practitioners that we will call the FPA (for Family Practitioner Association). All discussions of the FPA in this paper represent our own partial understanding of this case.

To earn a license to practice, freshly graduated medical doctors need to do six years of internships in domains related to their specialty. For the specialty of family practitioner, however, there is no specifically related internship. For example, a surgery intern might do 3 internships in surgery and 3 in other domains. An FP intern may do internships in 6 different domains. Selecting the right set of internships for FP interns is difficult. FPA members advise FP interns on the internships that will qualify them for a license in family practice.

The FPA has a list of interns who are interested in becoming FPs. To record information about these FP interns and available internships, the FPA uses a noncentralized, paper-based system.

The FPA secretary records on a spreadsheet the available internships and tracks the internships of each intern. The secretary connects the FP interns with FPA members who act as advisors. The advisors meet with the interns and explain which internships should be completed in order to qualify as an FP. The interns walk away from these meetings with a hand-written paper containing their personalized internship plan. During their internship period, the interns will meet with several advisors in different regions.

Mark explained that the previous president systematically compiled internship positions and reserved certain positions for FP interns. This represented a lot of work for his secretary and did not guarantee internships to all FP interns. Because of these difficulties, Mark decided to stop providing this service and focus on the advice given to FP interns concerning their internships. His goal is to improve the consistency of the recommendations given to the interns by the various advisors they meet during their internship period. He is therefore interested in a web-based system that FPA members and FP interns can use concurrently.

\section{An Overview of GORE Methods}

GORE methods use the concept of goal as the main construct for defining requirements. The use of the concept of goal in RE has emerged from research in software engineering where requirements began to supplant specifications as a way to describe the envisioned system. Researchers needed to analyze the environment of the envisioned system, because the system by itself could not guarantee the results expected by its stakeholders. The first papers linking goals and requirements date back to the beginning of the RE discipline, e.g. [9, 11, 27]. The seminal GORE paper was written by Dardenne et al. [10]. It introduced vocabulary inherited from Artificial Intelligence [20], e.g., goals, agents, roles, objectives, constraints, obstacles, and and/or graphs. Goals have been found to enable requirements engineers to: provide a higher-level and more stable view than requirements that implement them; to generate alternative solutions and select among them; and to analyze the requirements completeness and traceability [24]. 
Many GORE methods have been defined over the years. The most prominent are: KAOS, GBRAM [1], i* [37], GRL [13], TROPOS [18], ESPRIT CREWS [28], MAP [29] and Goal-oriented Use Cases [7]. Numerous goal types have been defined in these methods. The following is a partial list of goal types: achievement, maintenance, softgoal, feedback and satisfaction goals.

Goals very quickly became a central concept in RE. Zave and Jackson, for example, describe RE as, "Requirements Engineering is about the satisfaction of goals" [38]. Zave [39] defines RE as "the branch of software engineering concerned with the real-world goals for functions of and constraints on software systems." The call for papers of the Requirements Engineering conference series also strongly links requirements and goals, e.g. Requirements Engineering Conference 2004 [22]:

Requirements Engineering (RE) is the branch of systems engineering concerned with the goals, desired properties and constraints of complex systems, ranging from embedded software systems and software-based products to large enterprise and socio-technical systems that involve software systems, organizations and people.

\section{The Assumptions Behind GORE}

The focus on goals is understandable because it relates with the goal-seeking organizational model prevalent in the neighboring discipline of Information System [5]. Many GORE methods take for granted this goal-seeking model. Enterprise Modeling, for example, is said to include the goals of its agents [16] or members [21].

The base assumption underlying most GORE methods is that high-level enterprise goals can be gradually refined into requirements that can be assigned to the envisioned system $[10,31]$. This refinement is most often done with the help of and/or goal graphs inherited from [20].

Although many types of goals have been defined in GORE methods, the most popular goal type is the achievement goals; a goal that is to be achieved once and for all. The next most popular goal type is the softgoal. Both KAOS and GBRAM introduced the concept of maintenance goal, a goal that "is satisfied as long as its target condition remains true" (Anton and Potts, 1998). Maintenance goals have not received much attention and remain largely unused. This is particularly unfortunate because maintenance goals have been identified as "high-level goals with which achievement goals should comply" [2]. However, no explanation has been given to this high-level status of maintenance goals or to their relation with achievement goals.

\section{The Lack of Theoretical Grounding}

Several RE researchers have reported problems with the application of GORE methods, Goal discovery and goal refinement do not seem to be straightforward tasks. In particular, [1, 28. 38] note that "goals by themselves do not make a good starting point for requirements engineering." and that "Almost every goal is a subgoal with 
some higher purpose." They show that goal abstraction may lead to unrealistic or unwanted alternatives.

The proposed remedies are to bound goal abstraction and refinement with the subject matter of the organization [38], to use interview transcripts and organizational documents for goal discovery [1] and to use scenario and goal reasoning together so that they inform one another [28].

We believe that the problems identified by RE researchers are a sign of a deeper issue, necessitating a broader view of GORE and its relationships to individual and organizational behavior In their suggested roadmap for RE, Nuseibeh and Easterbrook [21] provide a very broad perspective on RE research. They explain that "RE is a multi-disciplinary, human-centered process"; that it uses cognitive and social sciences as theoretical grounding; and that [21]:

RE must concern itself with an understanding of beliefs of stakeholders (epistemology), the question of what is observable in the world (phenomenology), and the question of what can be agreed on as objectively true (ontology).

There has been very little theoretical grounding of GORE, and the three concerns identified by Nuseibeh and Easterbrook are missing in most GORE research. Questions such as the following receive little attention in this stream of research: what is the nature of goal-oriented behavior. Are goals the ultimate explanation of human and organizational behavior (as often assumed in RE)? Is there an explanation to the source of goals themselves (apart from scenarios, interviews and transcripts)? What is the relationship between goals, beliefs, observations and agreements?

\section{Goal Refinement and Abstraction}

Most GORE methods place goals in some hierarchy. Goal refinement is used to identify lower-level goals by asking how a given goal is achieved. Goal abstraction is used to identify higher-level goals by asking why a given goal needs to be achieved.

Although both why and how questions are encouraged by GORE methods, the how is much more prevalent in GORE publications. Most often, a so-called high-level goal is postulated to be strategic for the organization under analysis and is refined into subgoals. For example, van Lamsweerde gives the following examples for "highlevel, strategic concern": "serve more passengers" for a train transportation system" and "provide ubiquitous cash service for an ATM network system." [31]. It is not clear why these should be considered as high-level, strategic goals and how they can be satisfied. If the train transportation system serves a few more passengers, is this goal achieved? How many passengers are considered to be enough? Is there a limit to the number of passengers? Should the transportation system be designed to serve an infinite number of passengers? What will the system do next once this goal is achieved? What if this goal is never achieved? What would the ATM network system do once the ubiquitous cash service is provided? What are the criteria of achievement for a ubiquitous cash service? Similarly, $i^{*}$ highest-level diagram is called a "strategic dependency model" but nowhere is it explained what makes the goals expressed in this diagram strategic. 
Applying the same pattern to the FPA example, we would begin with the goal of maintaining advice consistency. We would then refine this goal into achievement subgoals: all advisors to an FP intern use FP intern record and Each FP intern uses own FP intern record. These goals in turn will be supported by system level goals such as, FP intern record available to FP intern advisors and FP intern record available to FP intern. Goal abstraction is even more difficult. To paraphrase Zave and Jackson [38]: what is the goal of maintaining the advice consistency? Is it to keep the FP interns comfortable? Is it to improve family practice? Shouldn't the goal of the FPA be to improve medical practice in general? To satisfy this latter goal, should the FPA consider to become the medical practice association (MPA)?

As can be seen in this example, no rationale is given to the goal refinement and abstraction. Why would the use of the same record guarantee the consistency of the advice? Should the FPA change to the MPA? In agent programming, this rationale is often provided with the use of beliefs. In KAOS, GBRAM, ESPRIT CREWS and MAP, goals are not embodied in an agent and they do not have a concept corresponding to a belief. In $i^{*}$ goals are embodied within agents and the concept of belief is defined, but is very seldom used.

\section{Survival and Regulation as the Source of Goals}

In this section we describe an epistemological view that explains the source of goals as emerging from the regulation mechanisms that are at the base of the survival of an organization in a changing environment. We present Vickers' appreciative system as a possible base for thinking about GORE methods.

\section{Survival as the Maintenance of Norms}

As we have seen, GORE methods seek to define the highest-level goals that are adequate for defining requirements for an envisioned system. Despite their important advancement, this is one aspect that the mainstream GORE methods (e.g. $i^{*}$, KAOS) have not defined yet. So-called high-level goals are often described as strategic goals in GORE papers. It is therefore important to understand what a strategic goal is. In the most general case, the highest-level goal that can be ascribed to an organization is to survive. We then have to define what we mean by survival. We have shown elsewhere, e.g. [24, 25], that survival can be understood in terms of the maintenance of an identity for a given observer. Maintaining this identity requires the maintenance of stable states within the boundaries defined by the observer [24]. These stable states are often called norms. Observers use the norms maintained by an organization to identify it as a separate entity from other organizations. Survival is therefore not an absolute measurement. It depends as much on the observer as on the observed. This means that some observers will select some norms as defining the organization and others will select other norms.

It is crucial that norms remain stable for an observer to recognize the organization over time. If a given feature of the organization changes its state beyond some 
threshold, the observer will not be able to identify it as the same feature as before the change and will therefore fail to recognize the feature and the organization. When two organizations merge, their norms become indistinguishable for observers.

The FPA, for example, has norms that separate it from other similar associations, such as, a unique name, mission, statutes, a logo and offices. It also has members, a president and a secretary. The individuals who fill these positions change over time, but relatively slowly. The FPA remains more or less the same (most of its norms remain the same) even when some individuals leave the association and some others join it.

\section{Regulation as a Source of Goals}

In a changing environment, organizational norms remain stable due to incessant effort to counter change $[35,36]$. This is often called regulation. Regulation is therefore a powerful source of action designed to bring a state closer to the norm. Norms also place constraints on possible actions by defining what is permissible and what is not.

The FPA attempts to maintain some norms that it deems important for the survival of the family practitioner practice, e.g. the number of family practitioners in its region, their level of expertise, their sense of community, their recognition among other MDs, among politicians and the general population. To maintain these norms, the FPA may take many actions, such as: presenting the FP practice to students; promote FP courses in the curriculum; provide advice to FP interns; Create FP communities in different regions.

\section{Organizations as Open Systems}

An organization needs energy in order to track the stability of norms, to spawn regulative actions when needed and to change norms when needed. In a closed system, energy is finite. When it is spent, the organization will lose its ability to maintain its norms and will disintegrate. The organization needs to have relationships with individuals and organizations in its environment in order to exchange energy with them and therefore have the means to maintain its norms. This is a consequence of the open system model of organizations [24]. These relationships must themselves be maintained within very specific threshold associated with a norm for the organization to be able to leverage them for maintaining its norms.

The FPA needs a continuous flow of FP interns either to replace retiring FPA members or to increase the number of FPs. The FPA also needs a steady stream of FP interns that it must convert into FPA members. FP interns must fit very stringent quality levels, which means that they need to be within the threshold range of the family practitioner norm. Internships must fit the tolerance range for qualifying interns for their FP license.

To promote the FP practice, the FPA has to be representative of this practice. This means that it has to insure that its members represent the majority of the FPs. The FPA needs as many members as possible, however, the members must be FPs or else the FPA will lose its FP identity. 


\section{Changing Norms to Fit the Environment}

The environment around the organizations continually changes and this induces changes to the organization's norms. Hence, to maintain its norms relatively stable does not mean that the norms do not change at all. Organizations that survive over the long-run make changes to their norms to fit the environment, but in a very controlled way. This means that changes must be maintained within the boundaries defined by its stakeholders in order for the organization to maintain its identity for these stakeholders.

The FPA remains the association for family practitioners. It does not become the association for surgeons or other specialty. However, it does change some of its norms over time. The new president is not interested in some of his predecessor's ways of operating, e.g., tracking and reserving internships, but is more concerned with the consistency of the advice given to FP interns.

\section{Vickers' Appreciative System and Goal Concepts}

A feedback regulator, for example, a thermostat or an automatic pilot, maintains a given state stable, the temperature or the course, by sensing the current state comparing it with the given state, and applying some action if the difference is above the tolerance level. Vickers $[32,33]$ proposes the concept of the appreciative system, by extending this model of a feedback regulation to human and organizational regulation. Vickers's appreciative system has three components [6, 26, 32, 33]: reality judgments, value judgments and action judgments. With reality judgments, some aspect of reality is singled out to be the study of attention. In value judgments, this reality judgment is matched to a category within which it is then compared to the norm (what ought to be). In Action judgment, some action might be taken to bring the reality judgment closer to the norm. The three judgments function as a complete system so that change to one of them requires change to the others. Hence, the way we view and judge the world affects our actions and our actions affect our view and judgments [32,33].

Action judgments are exercised when the reality judgment is considered to be outside the threshold associated with a relevant norm within the category in which the reality judgment was placed. This comparison brings about a host of debates about the course of action taken by the organization. For example, is the FPA promoting the FP practice enough, too much or too little? How important is the consistency of the advice? Should the FPA track the available internships? Is it useful to have a central registry of FP interns?

In a simple automaton the information to be sensed, the norm, the threshold to compare with and the kind of actions to be taken are all given by its designer. In an appreciative system, they are all subject to continuous change; nothing is set once and for all by a designer. Hence, an appreciative system creates its own dynamics, which an automaton does not.

Even though the appreciative system creates its own judgments, it is nevertheless a rather stable construct, i.e. it creates its own norms. Vickers calls these norms readiness. An individual or an organization has a readiness (a tendency) to see things 
in certain ways, to value them in certain ways and to act in certain ways. All these are rather stable in time. It is often the role of the analyst to try and shake-up these readinesses.

Vickers [33] insists on the fact that when a current state of affairs is outside of the tolerance level, no (externally visible) action is necessarily taken. It may very well be that either the reality judgments, the value judgments or both may change. Changing these judgments leads to the acceptance of the state of affairs rather than an attempt to change it. Likewise, action judgments can also change when the organization changes its behavior when dealing with similar situations. These changes to the appreciative system result in an adaptation of the organization to its environment. Changes to action judgments are much more visible because they result in changes to visible behavior. Changes to reality and value judgments are often much less visible,

This adaptation is visible in the FPA example. Recall that Mark revised his view of the FP intern problem during the project. He gave much less importance to the advice consistency problem and much more importance to the maintenance of a sense of community between FP interns and FPA members. In the appreciative system model, this means that he changed his value judgments. The reality judgment saying that the advices were inconsistent didn't change, but this inconsistency is now given much less value. However, maintaining the community is given much more value.

\section{Improving GORE Methods}

Based on the regulation view we have proposed in the previous section, we propose a number of improvements to GORE methods.

\section{Maintenance is Higher-Level Than Achievement}

As we have shown, the concept of maintenance goal is an approximation of the concept of norms, and norms relate directly to the survival of the organization for different stakeholders. Achievement goals model actions that are taken most often to maintain a related norm (modeled by a maintenance goal). This clarifies the status of maintenance goals as being higher-level than related achievement goals.

Mark's description of the system he was looking for made us focus on the following maintenance goal: Maintain the consistency of the advice given to FP interns. The lower level achievement goals can be: All advisors to an FP intern use FP intern record and Each FP intern uses own FP intern record.

\section{Maintenance Goals and Tolerance Levels}

The threshold associated with a norm defines the tolerance level of stakeholders. What they define as a problem or what they can live with. Maintenance goals are an approximation of norms because they lack the concept of tolerance levels. When does it become clear that a certain state does not satisfy the related maintenance goal? 
Maintenance goals should be augmented with tolerance levels so that they are better suited for modeling norms.

In the FPA example, this means that we need to augment the maintenance goal of maintaining the advice consistency with tolerances about the consistency. When are two advices considered to be inconsistent? How would different advisors consider the consistency of their advices?

\section{High-Level Goals and Alternatives}

Understanding that high-level goals model norms can help requirements engineering to seek the norms that different stakeholders consider as identifying the organization. This can lead to a more widespread use of maintenance goals, as the highest-level goals that model an organization survival are therefore necessary.

Identifying the norms that are considered essential for survival helps us solve the unacceptable goal alternatives identified by Zave and Jackson [38]. Identifying these strategic norms places the appropriate bounds on what alternatives are acceptable and not acceptable.

For the FPA, instead of simply taking the expressed goals of maintaining the consistency among advisors, it is interesting to understand what norms are essential for the survival of the FPA for different stakeholders. As we have seen, for Mark, the most important norm is the maintenance of a community of FPs. A higher-level goal to maintaining the advice consistency is therefore to maintain a sense of community between FP interns and their advisors. This goal can be refined into the maintenance goal of maintaining regular dinners between FP interns and advisors. This goal refinement is connected by beliefs as described below.

\section{The Appreciative system and GORE Concepts}

Linking the appreciative system and GORE aspects, we suggest to refine the framework we proposed in [24] by considering maintenance goals as a reflection of norms, beliefs can be as a reflection of reality and value judgments, and achievement goals as a reflection of action judgments.

From this point of view, GORE methods have mainly concentrated on the third stage, action judgments (achievement goals), and neglected the other two, norms (maintenance goals), and reality and value judgments (beliefs). This is easily understandable, if we consider that action judgments are much more visible than reality and value judgments.

Considering beliefs as reflecting reality and value judgments, means that beliefs should become a major concept in GORE methods. Because the three judgments of Vickers' appreciative system are interlocked, goals cannot be changed without changing the beliefs that justify them. This means as much as possible, stakeholders goals must be justified by corresponding beliefs. Alternative goal refinements (i.e., the or part of the and/or graph) must be justified by different sets of beliefs. Goal abstraction, likewise, leads to beliefs rather than directly to higher-level goals. 
For the FPA, asking Mark why he wants a centralized system elicited the answer that FP interns were receiving inconsistent advice from the advisors they met. Mark believed that this inconsistency might distance FP interns from the FP practice and from the FPA. A relevant higher-level goal is to keep the FP interns within the FPA community. A subgoal is to maintain a sense of community between FP interns and their advisors. The underlying belief is that a sense of community keeps people together). Mark has now the subgoal of putting in place community building mechanisms, such as regular dinners bringing together FP interns and their advisors. The underlying belief is that these regular dinners will strengthen the sense of community between the FP interns and their advisors and will therefore reduce the risk of FP interns leaving the FP practice because of the inconsistency of the advice they receive.

The following example shows other goals that were influenced by beliefs during this project. To stop tracking internship information, Mark and his secretary must believe that it is not necessary anymore. An interview with the secretary showed that she believed that tracking available internships was still necessary, even though Mark believed that it was not.

\section{Related Work}

Several conceptual studies of GORE methods have been published over the years, e.g. $[14,15]$. These studies assume a viewpoint from within the RE research paradigm. They do not ground their research in an external body of knowledge, which limits their explanatory power of goals. In recent years, $i^{*}$ has become the main GORE method. Much research has applied i* in different contexts. Recently, a well received study of the $i^{*}$ graphical notation was published [17], noting that major improvements are needed in order to make $i^{*}$ user friendly. The study, however, was limited to the graphical level and did not investigate the epistemological or ontological aspects of $i^{*}$.

Our work is similar in nature to Checkland and Holwell's conceptual cleansing [5] of the field of information systems. Checkland [4] has worked extensively to popularize Vickers's work with Soft System Methodology (SSM). Ours is a very short description of Vickers's appreciative system. More elaborate descriptions are available in Vickers's writings [32, 33], and in [4, 6, 26]. We proposed an explanation of goals based on General Systems Thinking and Vickers's work in [24].

Sutcliffe and Maiden [30] proposed a notable kind of goal in a paper that seems to have received little attention by GORE researchers. They proposed 6 classes of goals. One of the classes is called "feedback goals." They describe these goals as maintaining a desired state with a related tolerance range, spawning corrective actions when the state is considered to be outside the tolerance range [30]. This class of goals seems to have gone unnoticed by subsequent GORE research. We have ourselves added feedback into GORE research about 10 years later [23, 24] without noticing the significance of Sutcliffe and Maiden's feedback goal class at the time. 


\section{Conclusions}

RE is about understanding peoples' desires and maybe designing some automated system to help them to obtain or maintain them. RE must therefore find the balance between what is desired and what is feasible. To understand what is desired, it is necessary above all to understand individual and organizational behavior. GORE methods have made major contributions to the practice of RE but have modeled this behavior in too simplified terms, mostly as goals to be achieved. In this paper, we have shown that goals can be seen as the visible part of regulation. Regulation models the way individuals and organizations attempt to survive in a changing environment.

Regulation results in the establishment of norms, stable states that define the identity and therefore the survival for a given observer. A long lasting organization manages its internal and external relationships in a way that controls the changes to these norms but still allows them to change when needed. Looking at regulation rather than goals shifts the attention to the way people manage stability and change by managing relationships.

GORE methods already have useful concepts needed for the study of regulation, e.g. maintenance goals, and beliefs. These concepts need to be used much more than they have been until now and they need to be extended with more regulation concepts. We have shown some of these concepts, e.g., the threshold that defines the tolerance of deviations from a norm, reality judgments and value judgments. Obviously, much more research can be done on modeling reality and value judgments as beliefs. Many regulation concepts are described in [35 and 36]. It will be useful to add them to GORE methods.

In this paper we limited our epistemological discussion to Vickers' appreciative system. There are many other conceptual frameworks that can be used, for example, Weick [34] and the Viable System Model (VSM) [3].

\section{References}

1 Anton, A.I.,: Goal-based requirements analysis. Proc. ICRE'96 Second International Conference on Requirements Engineering, IEEE (1996)

2 Anton, A.I., Potts, C.: The use of goals to surface requirements for evolving systems. In ICSE 98 International Conference on Software Engineering, IEEE, (1998)

3 Beer, S.: The viable system model: its provenance, development, methodology and pathology. Journal of the Operational Research Society, 35(1), 7-25 (1984)

4 Checkland, P.: Soft System Methodology: a 30-year retrospective Wiley, Chichester UK (1999)

5 Checkland P, Holwell, S.: Information, systems and information systems - making sense of the field. Wiley. Chichester, UK (1998)

6 Checkland, P: Webs of significance: the work of Geoffrey Vickers. Systems Research and Behavioral Science, 22:4, Wiley (2005)

7 Cockburn, A.: Writing Effective Use Cases, Addison-Wesley. Reading, MA (2001)

8 Constantine, L.: Essential modeling: Use cases for user interfaces. In ACM Interactions, 2(2), 34-46 (1995) 
9 Dardenne, A., Fickas, S., van Lamsweerde, A.: Goal-directed concept acquisition in requirements elicitation. In IWSSD '91, Sixth International Workshop on Software Specification and Design, ACM (1991)

10 Dardenne, A., van Lamsweerde A., Fickas, S.: Goal Directed Requirements Acquisition. Science of Computer Programming, 20(1-2), 3-50 (1993)

11 Dubois, E.: A Logic of Action for Supporting Goal-oriented Elaborations of Requirements. In IWSSD '89, Fifth International Workshop on Software Specification and Design, ACM (1989)

12 Gause, D.C., Weinberg, G. M.: Exploring Requirements: Quality BEFORE Design, Dorset House, N.Y. (1989)

13 ITU-T, Telecommunication Standardization Sector of ITU: User requirements notation (URN) - Language definition (Z.151) (2008)

14 Kavakli, E.: Goal-Oriented Requirements Engineering: A Unifying Framework. Requirements Engineering, 6(4), 237-251 (2002)

15 Kavakli, E., Loucopoulos, P.: Goal Modeling in Requirements Engineering: Analysis and Critique of Current Methods. Information Modeling Methods and Methodologies, 102124 (2005)

16 Loucopoulos, P., Kavakli, E.: Enterprise Modelling and the Teleological Approach to Requirements Engineering. Intelligent and Cooperative Information Systems, 4(1), 45-79 (1995)

17 Moody, D., Heymans, P., Matulevičius, R.: Visual syntax does matter: improving the cognitive effectiveness of the $i^{*}$ visual notation. Requirements Engineering, 15(2), 141$175(2010)$

18 Mylopoulos, J., Kolp, M., Castro, J.: UML for Agent-Oriented Software Development: The Tropos Proposal. In UML 2001, Fourth International Conference on the Unified Modeling Language, Springer (2001)

19 Mylopoulos J.: Goal-Oriented Requirements Engineering: Part II. Keynote Talk, RE'06, 14th IEEE Requirements Engineering Conference, Minneapolis, MS, September 2006. http://www.ifi.uzh.ch/req/events/RE06/ConferenceProgram/RE06_slides_Mylopoulos.pd f, accessed May 2011 (2006)

20 Nilsson, N.J.: Problem Solving Methods in Artificial Intelligence, McGraw-Hill (1971)

21 Nuseibeh, B., Easterbrook, S.: Requirements engineering: a roadmap. In ICSE '00, International Conference on The Future of Software Engineering, ACM (2000)

22 RE04, 2004. Requirements Engineering International Conference 2004, http://www.re04.org/, accessed May 2011 (2004)

23 Regev, G., Wegmann, A.: Defining Early IT System Requirements with Regulation Principles: the Lightswitch Approach.. Proc. 12th IEEE International Requirements Engineering Conference (RE'05), Kyoto, Japan (2004)

24 Regev, G., Wegmann, A.: Where do Goals Come From: the Underlying Principles of Goal-Oriented Requirements Engineering. Proc. 13th IEEE International Requirements Engineering Conference (RE'05), Paris (2005)

25 Regev, G. Gause, D.C., Wegmann, A.: Creativity and the Age-Old Resistance to Change Problem in RE. Proc. 14th IEEE International Requirements Engineering Conference (RE’06), Minneapolis, MN (2006).

26 Regev, G., Hayard, O., Wegmann, A.: Service Systems and Value Modeling from an Appreciative System Perspective. In IESS1.1, Second International Conference on Exploring Services Sciences, Springer (2011)

27 Robinson, W. N.: Integrating multiple specifications using domain goals. In In IWSSD '89, Fifth International Workshop on Software Specification and Design, ACM (1989)

28 Rolland, C., Souveyet, C., Ben Achour, C.: Guiding goal modeling using scenarios. IEEE Trans. Software Eng., 24, 1055-1071 (1998) 
29 Rolland, C., Salinesi, C.: Modeling Goals and Reasoning with Them, Engineering and Managing Software Requirements (EMSR), A. Aurum, C. Wohlin (editeurs), Springer Verlag, (2005)

30 Sutcliffe, A.G., Maiden, N.A.M.: Bridging the Requirements Gap: Policies, Goals and Domains. In IWSSD '93 7th International Workshop on Software Specification and Design, IEEE (1993)

31 van Lamsweerde, A. Goal-Oriented Requirements Engineering: A Guided Tour, in RE'01, 5th IEEE International Symposium on Requirements Engineering, IEEE (2001)

32 Vickers, Sir G.: Value Systems and Social Process. Tavistock, London (1968)

33 Vickers, Sir G.: Policymaking, Communication, and Social Learning. eds, Adams, G.B., Forester, J., Catron, B.L., Transaction Books. New Brunswick NJ (1987)

34 Weick, K. E.: The Social Psychology of Organizing, second edition. McGraw-Hill (1979)

35 Weinberg, G. M.: An Introduction to General Systems Thinking. Wiley \& Sons. New York (1975)

36 Weinberg, G.M., Weinberg, D.: General Principles of Systems Design. Dorset House, New York (1988)

37 Yu, E.S.K.: Towards modelling and reasoning support for early-phase requirements engineering. In RE'97, Third IEEE International Symposium on Requirements Engineering, IEEE (1997)

38 Zave, P. and Jackson, M.: Four Dark Corners of Requirements Engineering, ACM Transactions on Software Engineering and Methodology, 6(1), 1-30 (1997)

39 Zave, P.: Classification of Research Efforts in Requirements Engineering, ACM Computing Surveys, 29(4), (1997) 